\title{
Altering User Movement Behaviour in Virtual Environments
}

\author{
Category: Research
}
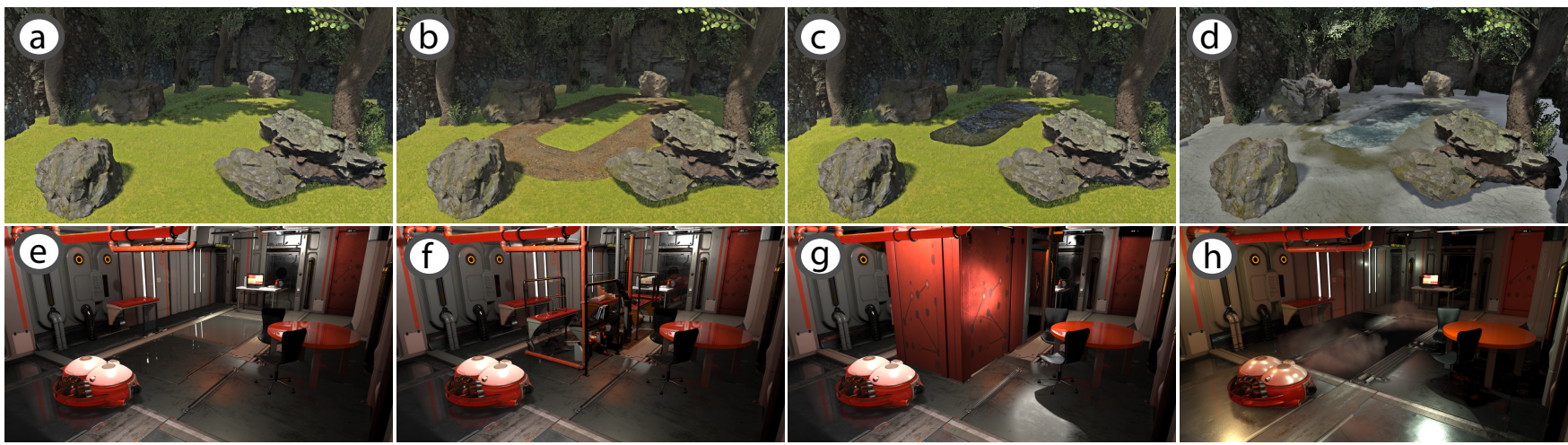

Figure 1: Above, the two sets of Virtual Environments we designed for the study. These environments are based on a physical environment, where each object is paired to a physical proxy. The first row shows alterations of the representation of the central area through different surfaces. The second row introduces different types of immaterial obstacles in the central area. Additionally, a virtual replica of the physical environment was created (see Figure 2).

\begin{abstract}
In immersive Virtual Reality systems, users tend to move in a Virtual Environment as they would in an analogous physical environment. In this work, we investigated how user behaviour is affected when the Virtual Environment differs from the physical space. We created two sets of four environments each, plus a virtual replica of the physical environment as a baseline. The first focused on aesthetic discrepancies, such as a water surface in place of solid ground. The second focused on mixing immaterial objects together with those paired to tangible objects. For example, barring an area with walls or obstacles. We designed a study where participants had to reach three waypoints laid out in such a way to prompt a decision on which path to follow based on the conflict between the mismatching visual stimuli and their awareness of the real layout of the room. We analysed their performances to determine whether their trajectories were altered significantly from the shortest route. From the results obtained and our observations, we derive guidelines on how to alter user movement behaviour in Virtual Environments.
\end{abstract}

Keywords: Virtual Reality; Locomotion; User Behaviour.

Index Terms: Information Interfaces and PresentationArtificial, augmented and virtual realities

\section{INTRODUCTION}

Exploring Virtual Environments (VE) by walking is the most realistic and natural interface available to Virtual Reality (VR) systems where users embody anthropomorphous characters [38]. It is also the most technically challenging interface due to hardware issues (tracking systems, instrumentation that needs to be worn) [3] and environmental issues (disparity between the extents of the VE and the physical space available [18]; existence of physical elements not present in the simulation [23]). While various solutions to these problems have been presented [18, 30, 37], these are not always adaptable to domestic environments such as homes, offices and any other location not explicitly designed as a VR laboratory. Consequently, current home-VR applications require users to be seated or move within confined areas to mitigate the problem.
We think that domestic VR represents a fundamental stepping stone for its widespread adoption. However, these environments provide different challenges in comparison to VR laboratories: in homes, furniture constrains the navigable space. Instead, VR labs are usually large and empty rooms. Incorporating objects from the user's immediate physical surroundings into the simulation, by substituting them with mismatching virtual counterparts, has the potential of providing a compelling VR experience [22, 23]. However, where past work has focused on locomotion within matched environments [5] or empty environments [18], less attention has been given on whether altering the mismatch between the physical environment and its virtual representation affects users' movement behaviour. If so, are there specific alterations that are more impactful than others?

In this paper, we studied two types of alterations to the representation of the VE: 1) aesthetic mismatch in the representation of surfaces; 2) mixing virtual objects that are paired to a physical proxy together with immaterial ones that are not. We asked 18 participants to walk towards a sequence of three virtual objects which were paired to physical objects. We designed four outdoor (with different surfaces) and four indoor VEs (with immaterial objects) with an increasing level of mismatch from the physical room (see Fig. 1) used as a baseline. Our goal was to evaluate how those two factors could alter user's movement behaviour. We know that walking trajectories in VR tend to conform to those performed through natural locomotion [3]. Thus, we placed these waypoints in such a way that the shortest, most direct, route went through an area that prompted a choice between adhering to the visual stimuli and thus taking a longer route, or ignoring it in favour of their awareness of the physical room. For example, in one of the outdoor VEs, this area was represented as a water pond, while in one of the indoor VEs it was surrounded by walls. This allows us to understand how their behaviour is affected and thus, how to influence it.

Being able to influence user movement behaviour can have several beneficial applications. For example, VR cinematographers could maximise the chances of users moving through specific areas, in order not to miss the action. In domestic VR we might want to avoid walking near fragile objects or near our sleeping pet. If alter- 
ations to the aesthetic design are sufficient to subtly encourage users to take a specific path, or conversely, deter them from taking another one, we can minimise the impact of such situations arising in a way that does not affect the believability of the experience [17]. Analogously, understanding how to alter movement behaviour by placing immaterial objects is essential to portray VEs that have significant differences in their extents, compared to the physical environment. For example, a virtual hallway and a real living room.

Our results indicate that both the aesthetic appearance of surfaces and immaterial virtual objects do affect user trajectories. We found that surfaces elicited different behaviours drawn from reallife experiences, depending on the adverse consequences users expected by walking over. However, if users are not able to interpret the visual stimuli as intended, altering the appearance of a surface loses its effectiveness. Immaterial objects, instead, were the more likely to affect users' trajectories in deviating from the shortest route. Immaterial objects helped participants focus their interactions where the tangible ones were located. Participants also reported that the combination of immaterial and tangible objects reinforced the illusion that they might all really be tangible.

\section{Related Work}

Locomotion is a quintessential feature in VR. In many immersive application scenarios users need to be able to change their position and viewpoint. This is achieved by means of "locomotion interfaces", that is, an interaction technique that, through an underlying system, is able to map user input into movement in the VE. Due to the wide spectrum of 3D applications, these interfaces can be differentiated by the fidelity with which they simulate the act of walking. In the following, we describe the main locomotion interfaces and studies that investigated movement behaviour.

\subsection{Natural Walking}

High-Fidelity techniques are those that most accurately reproduce the act of walking. Especially in those scenarios where users are embodied by a virtual character, really walking in a physical space is the most natural choice. Natural walking usually refers to scenarios in which the VE is completely contained within the physical space where the VR system is deployed. In these circumstances, one step in the real world corresponds to a step of equal length in the VE. This locomotion interface has been found to be a presence-enhancing factor [38] when compared to lower fidelity locomotion techniques such as Walking-in-Place [37] or virtual flying [1]. Natural walking has also been found to result in less obstacles collisions [20], to require less training time [21], to more closely conform to real-world trajectories [40] or behaviour [36], and to retain more accurate information about the environment traversed [44].

\subsection{Redirected Walking}

In those scenarios where users are required to explore VEs that are larger than the physical space available, natural walking interfaces are less suitable. "Redirected Walking" refers to a category of techniques that allow users to physically explore virtual spaces larger than their physical surroundings, by altering how the physical path in the real world is perceived in the VE [18].

Suma et al., presented a taxonomy of redirection techniques [35], extending prior work by Steinicke et al. [34]. The authors classify these techniques according to whether they affect translation or rotation, on the extent of the alteration (discretely or continuously), and whether subtle or overt. Examples of subtle techniques that affect the amount of space travelled by users are the "Seven Leagues Boots" (continuosuly) [12] and a technique by Bruder et al. that compensates distance perception through self-movement illusions (discretely) [2]. Overt continuous techniques couple the translational change with navigation facilitators, such as vehicles or elevators [9]. Discrete techniques use well known sci-fi metaphors of portals to navigate between different parts of an environment $[6,31]$.

Reorientation techniques affect the user's viewpoint in the world. Subtle techniques include the work of Razzaque et al. [18] on Redirected Walking, which continuously introduce non-noticeable gains to head rotations, so that users can explore larger than physical spaces. These alterations have been shown to be tolerable when within thresholds $[32,33]$. However, a drawback is the requirement of a large spatial radius to avoid becoming noticeable. This radius has been reported to be at least $22 \mathrm{~m}$ [10], to more than $40 \mathrm{~m}$ [32]. This makes these techniques unsuitable for smaller domestic environments. Discrete techniques instead rearrange the location of some architectural features such as doorways. However, this strategy is unsuitable for non-abstract environments where maintaining a coherent spatial relationship is key. Overt continuous techniques explicitly require users to reorient themselves upon reaching the boundaries of the environment [42]. Distractor elements, objects, or characters that attempt to catch the user's attention by forcing them to turn their head, provide an opportunity to introduce these gains while the user is turning to face the stimulus [17]. Discrete techniques instead explicitly require users to change their heading while freezing the tracking updates [42].

\subsection{Walking-in-Place}

Walking-in-place (WIP) is a category of techniques where, as their name implies, the user's stationary gait is mapped to movement in the VE $[28,37]$. A redirected version of WIP keeps users from facing the absent back wall in a three-sided CAVE setup [19]. WIP is typically implemented via sensors or infra-red markers, but it was also demonstrated on a Wii Balance Board [41] and by detecting feet shadows on the floor [45]. WIP has been reported as being less realistic than natural walking, and to result in problems related to latency and lack of smoothness during movement [4].

\subsection{Abstracted walking interfaces}

"Virtual Flying" is among the most common and widely employed techniques where manipulation of the viewpoint occurs through an input device, such as a joystick or mouse and keyboard interface [1]. Another variant of this concept, the "Human Joystick", is a technique developed by McMahan et al. for use in CAVE systems where the position of the user in respect to the centre is used as a movement vector [15].

\subsection{Treadmills}

Omnidirectional treadmills support walking in any direction [30]. However, while enhancing the virtual navigable space and providing a better simulation of the act of walking, their size would require radical rearrangements of the domestic environments in which these are going to be used. Treadmills also do not simulate uneven terrain or obstacles.

\subsection{Locomotion Behaviour}

Studies in this domain have mainly focused on performance metrics, where different locomotion interfaces are compared. Metrics include task completion time, number of collisions, cognitive recall, presence, etc. Differently from the works previously cited, here we investigate factors affecting the behaviour adopted by users while walking in a VE. Other researchers have studied locomotion behaviour by analysing the trajectories taken by users. Fink et al. [5] found that obstacle avoidance trajectories differ only slightly between a VE and a matched real environment. In unconstrained goal-directed tasks, trajectories in VE conform to those in a real-world version of the task [3]. Ruddle et al. [20] found that natural walking influenced users to walk around obstacles, whereas 
lower fidelity interfaces had a greater incidence of obstacle interpenetration. Cirio et al. [3] presented a framework of trajectographical criteria that compares the realism of virtual against real trajectories. The framework includes nine metrics related to the shape, performance, and kinematic features. Other researchers have used similar metrics: area between virtual and real trajectory, time-based or curvilinear distance, and gait-based parameters.

One of the most famous reports of a mismatching VE influencing participant trajectories, is the "Pit Room" [11]. In this VE, passive haptics in the form of a raised ledge, gave participants the illusion of standing next to a pit. Consequently, their trajectories followed the ledge. The main focus of this work was investigating whether the illusion was convincing. In our work, we are interested in understanding the behaviour of users when forced to make a navigational choice based on the conflict between the visual stimuli of the VE and their knowledge of the real space they are in.

\section{USER StUdy}

The goal of the study was to investigate how different types of alterations in the design of a VE affected movement behaviour. We considered two broad alteration categories: the aesthetic design of the environment's surface area (studied in a set of four outdoor VEs) and the impact of immaterial objects mixed with physicallypaired virtual objects (studied in a set of four indoor VEs). Each of the individual VEs presents a type of alteration (detailed in the following) we believed would be generalisable to a range of similar scenarios. In a real world scenario, a VE designer could conceivably use multiple types of alterations together. We studied them independently from each other to analyse their individual impact on user movement behaviour.

\subsection{Task}

The task required participants to follow a counter-clockwise route between three specific locations in the room the study took place. These locations, or waypoints, were arranged in such a way that one of the legs of the shortest route went directly over the central area. At each waypoint, we placed a real furniture item (a table, a shelf unit, and a desk). Participants had to perform three laps in each environment. Upon reaching each waypoint, we instructed participants to touch the object they saw (the actual furniture item in the real word/replica conditions, or the substitutive object they perceived in VR) before continuing to the next one. This was intended to simulate interactions happening in the area. Upon reaching the last waypoint, we told them to go back to the first, touch the object again, and wait for our signal before starting the next lap.

Figure 2 shows the triangular shortest path between the three locations. We asked participants to go back towards the first waypoint (the table), in order to force them to deal with the experimental conditions. Each VE was altered in such a way that the most realistic route would have avoided the central area, thus deviating from the shortest direct route. Participants started the first lap from the couch (waypoint 0 ), as we decided not to have a new training phase before each new environment. After pilot testing, we observed that the distance covered before reaching the initial waypoint to be sufficient in helping participants to get their bearings, before actually starting the task. Furthermore, as explained in the Analysis section, the trajectographical data is only collected between the points of closest approach to the initial waypoint, filtering out further movements.

\subsection{Virtual Environments Design and Rationale}

The design of each VEs responds to the study goal of identifying which alteration types had the most impact on users' movement behaviour. The principle behind the design of each VE was to choose a "baseline" environment and create "altered" environments by changing the central area (or its immediate surroundings), while keeping everything else unchanged. This central area was $3.5 \mathrm{~m}$ long and $1 \mathrm{~m}$ wide. There was a clearance of $1 \mathrm{~m}$ between the perimeter of the central area and the objects. In the physical location where the study was conducted, this area was free from obstacles. We did not include any visual indicator such as markers or "gates" [3] in the design of VE, because we thought they could influence users' behaviour. We then identified examples of alterations, within the two categories, that might have the potential of affecting users' trajectories. In addition, we designed a replica of the room where we performed the experiment to represent a baseline condition (see Figure 2). All environments were enriched with ambient sounds according to each VE's unique characteristics.

The first alteration category focused on the aesthetic mismatch between the real surface and the virtual surface. We chose an outdoor "theme" for the four related environments, as in the outdoors it is not uncommon to find different surfaces in a limited area. We created a baseline outdoor Substitutional Environment [23] consisting of a grassy area. The walls of the room and the furniture were substituted by trees and rocks of similar size (see Figure $1(a)$ ). The three alterations we made, either directly to the surface used in the central area or in its external borders, are representative of different walking comfort levels. The path VE $(b)$ altered the baseline environment by suggesting a route around the central area reminiscent of nature trails. The water VE (c) substitutes the central area with a shallow pond. Walking over it in real life would be considered as a possible inconvenience if not explicitly necessary. Finally, the ice VEs $(d)$ substitutes the central area with an icy surface and the remainder with contextually appropriate icy grass. Walking over ice in real life would have a risk of slipping.

The second alteration category focused on the presence of various types of immaterial obstacles coexisting with virtual objects paired to physical proxies. The concept which informed the choice of the specific alterations was to have a gradual increase in the difficulty of overcoming those obstacles considered as having the potential to affect user trajectories. We chose an indoor theme for these VEs, as we believe obstacles would be more appropriate in such a setting. We created a Substitutional Environment consisting of an open plan room where the walls, floors and furniture were substituted by similar objects from the indoor assets available (see Figure 1(e), the baseline indoor VE). The objects VE $(f)$ altered the baseline by placing immaterial objects in the central area. While blocking access through the shortest route, users could still pass through the gaps between objects. The walls VE $(g)$ instead completely surrounded the central area by walls. We added a further dark VE $(h)$ where the immaterialness was subtractive rather than additive. We altered the floor and the lighting of the environment so that the central area appeared completely dark, with steam rising from the floor $(h)$.

We wanted to understand how and why participants decided to take the route they chose. We know that users tend to avoid parts of the VE they perceive as obstacles, as evidenced by studies on obstacle avoidance [5]. However, there is little information on how effective each alteration type is at influencing user trajectories. If some participants decided to walk over the water pond or through a wall, we sought to understand the reason behind this decision. Analogously, we wanted to understand why others instead decided to detour to avoid a non-existing obstacle. With this knowledge we will be able to understand how to influence user behaviour in VEs. Further, observing actions that are not mirrored exactly in the same way in reality (such as not experiencing a physical collision when walking through a wall in VR), will give us insights on how we can make this misalignment more convincing. 

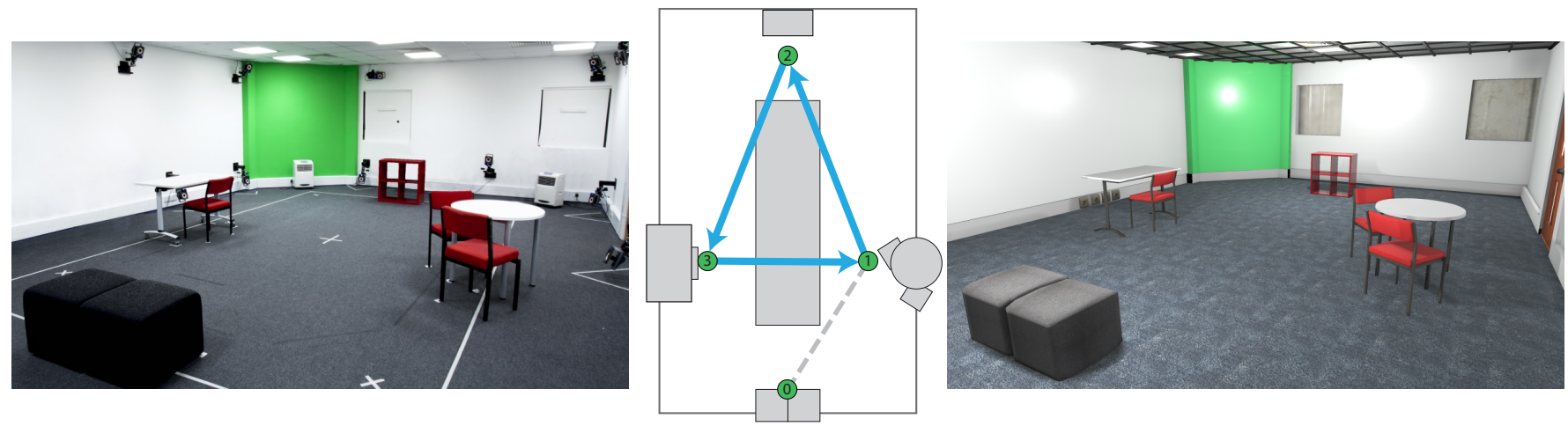

Figure 2: On the left, a picture of the VR laboratory. In the middle, the figure shows the layout of the room. The black border represents the reliable capture area. Some items were pushed outwards to allow for enough space to comfortably walk in those environments where the central area (in grey) appeared blocked. The blue path represents the shortest distance between waypoints. On the right, the virtual replica of the room.

\subsection{Participants}

Eighteen participants (10 male, 8 female) aged 20-45 $(M=$ $30.06, S D=7.55)$ took part in the study. We asked them to rate their experience with VR technologies and computer games in general, on a scale of 1 (rarely use VR technologies/play games) to 7 (very frequently use VR technologies/play games). Our participants had low experience with VR technologies $(M=2.56, S D=1.62)$ and average experience with games $(M=4.72, S D=1.90)$. Each participant was compensated by a $£ 10$ voucher for their time. Each study session took approximately 75 minutes.

\subsection{Procedure}

After signing a consent form describing the task, the investigator asked participants to confirm their demographics details. We then asked participants to reach each group of objects while walking in the most natural way possible. We explained that we wanted to study their movement behaviour, without mentioning the specific metrics used.

Participants performed three laps for each of the 12 environments we considered. To establish a baseline, all participants first performed the task in the real world (while wearing the headset on top of their heads, to simulate similar conditions as those for the other VEs). Successively, they repeated the task in a virtual replica of the room. The four outdoor and four indoor environments were then presented in counter-balanced order. Nine participants experienced the outdoor environments first and nine other experienced the indoor ones first. Furthermore, the order of presentation within each set was also counter-balanced. After these 8 VEs and before concluding the study, we asked participants to repeat the task in the virtual replica first and then in the real world. This allowed us to evaluate whether there were differences between the initial and final trajectories in the real world and in the replica VE.

In between each session (except the last repeated two) we asked participants to fill an SUS questionnaire [29] on presence. In addition, we asked them three further questions on a 7-point scale to ascertain: 1) how similar they felt the experience of walking in the environment was compared to the idea of walking in a similar environment in reality ( 1 - completely different, 7 - exactly the same); 2 ) if they felt engaged or distracted to the idea of actually walking on that surface ( 1 - completely distracted, 7 - completely engaged); 3 ) whether the environment affected their movement behaviour (1 - not at all, 7 - completely). All questions were asked while the participants were resting (with the headset removed) on the couch. Finally, for each environment, we conducted a semi-scripted interview to elicit feedback from their experience.

\subsection{Apparatus}

Here, we describe the physical room in which the study took place, the technical framework used to design the nine virtual spaces, and the equipment used by our participants.

The physical space - The study took place in a room where 20 Vicon T10 series cameras were installed on the walls, managed by the Vicon Blade 2.6.1 software. They were set to capture at 60 frames per second. The volume of reliable capture was $6.3 \mathrm{~m}$ long, $4 \mathrm{~m}$ wide, with a ceiling height of $2.5 \mathrm{~m}$. In the physical space, eight objects were placed at perimetral locations of the capturing area (see Figure 2): two small couches (0), a table with two chairs (1), a small shelf unit (2), and a desk with chair (3). We measured the dimensions of all objects as well as the room's extents. These measures were used to adapt the objects substituting their real counterparts and to model their virtual replicas.

The virtual space - We designed the nine VEs using Unreal Engine 4.6.1. One of the VEs was a replica of the physical room and the furniture used. The assets used in the other VEs were adapted from the freely available "Open World" and "Sci-Fi Hallway" collections in the Unreal Engine.

The user - Participants wore an Oculus Rift Development Kit 2 (DK2) headset. We installed four optical markers, one attached on the front-center, and the rest on the sides and behind. These provided absolute positioning and orientation data for the user's viewpoint in the VEs. Using the internal DK2 latency tester, we measured an average latency of $30 \mathrm{~ms}$. Since the DK2 cannot be used in a wireless configuration, we used a $10 \mathrm{~m}$ HDMI $1.4 \mathrm{~b}$ cable together with a similarly long USB extension, to avoid encumbering participants with a backpack. During the study, one of the investigators followed the participant, making sure that the cables did not hinder their movements.

To support a sense of proprioception, participants also wore a pair of fingerless gloves with a combination of four optical markers each attached on top. The markers were mapped to the position and orientation of two virtual hand models that the user could see in the VEs. We recalibrated all cameras and the user's virtual hands daily. Since each tracked "bone" puts additional strain on the rendering performance, we were not able to also track the users' feet as the frame rate decreased below acceptable levels (Sony advises a minimum of 60 FPS [16]), with the hardware at our disposal (an MSI GS70). Therefore, we prioritised hands because the task involves touching, whereas feet are not always in view.

\subsection{Analysis}

In order to investigate how the VEs affected the participants' movement behaviour, we used a set of quantitative metrics described by Cirio et al.'s [3]. The raw trajectories were first filtered by 
extracting only the part between the points of closest approach to the first waypoint, when beginning the lap and when returning back. Thus, the initial approach in the first lap and any further movement after completing the lap and returning to the first waypoint are filtered out. The filtered data for each lap are then considered as successive repetitions.

Initial assumptions - We compared the trajectographical results of the two baseline environments in each set (Outdoor Grass and Indoor Base) to the four VR laboratory environments (Real World: Start and End; Replica: Start and End) to confirm the assumption that virtual trajectories conform to trajectories performed in matched (or similar) physical environments [3, 5]. Successively, we compared the results of the three altered VEs to the associated baseline VE.

Trajectographical metrics - These metrics give information about the shape of the trajectories performed by participants. By comparing the values of the altered VEs to the baseline VE, we can determine where a significant departure from the shortest trajectory occurred. These measures are calculated on the filtered trajectories. Deviation: filtered trajectories were resampled through a set of 100 equidistant points. For each point, the absolute value of the perpendicular distance to the closest segment was taken (in centimetres). We expected that higher significant values in this criteria would show which environments impacted participants' trajectories the most.

Area of Deviation: by calculating the perpendicular distance at each resampled point, it was also possible to calculate the extents of the area representing the difference between the participant's trajectory and the reference triangular shortest path (in squared metres).

Curvature: for each sample, we also calculated the mean radius of curvature of the trajectory [5]. This quantity (in metres) gives a measure of how much the trajectory deviates from a straight line.

Kinematics data - We also calculated kinematics data such as the total distance covered (in metres), the time each lap took (in seconds; again, automatically calculated on the filtered trajectory) and the average speed during the motion (in centimetres per second).

\section{Results}

We conducted a repeated-measures one-way ANOVA (GreenhouseGeisser corrected where necessary) using the type of alteration in each VE as our independent variable, and the previously described metrics as our dependent variables. For each participant, we collected 12 Alteration $\times 3$ Repetitions trials, for a total of 648 . Of these, four $(0.6 \%)$ were missing due to logging system failures, leaving 644 complete trajectories. After each environment (apart from the final assessment of the real world laboratory and its VR replica) we asked our participant to fill an SUS questionnaire [29] on presence, and to answer three additional questions previously described in the Procedure section.

The results (summarised in Table 1 and Table 3) confirm that while both the presence of immaterial objects and the design of the surface have a significant impact on users' movement behaviour, the former had a stronger impact.

\subsection{Initial Assumptions}

In terms of quantitative measures, we did not find any significant difference between the Real World, the Replica, and the two altered baseline environments (Outdoor and Indoor Base). Mean deviation in these VEs are in the range of $22-28 \mathrm{~cm}$ (see Table 1). Visual inspection of the trajectories supports the initial assumption of our analysis, that trajectories in virtual replicas of a physical environment tend to conform to real trajectories, as found by Fink et al. [5] and Cirio et al. [3]. We can extend this result by noting that virtual trajectories tend to conform to real ones even in VEs that substitute all physical objects with mismatching virtual counterparts of approximately the same size.

No differences were also found between the initial and final assessment of the Replica and the Real World apart from their speed: participants were significantly faster $(M=90.34 \mathrm{~cm} / \mathrm{s})$ during the final Real World trials than in the initial VR Replica ones $(M=75.28 \mathrm{~cm} / \mathrm{s}$, accountable by the increased confidence accrued during the rest of the study.

\subsection{Trajectographical measures}

We found a significant effect of alteration for all trajectographical metrics: Deviation $\left(F_{11,187}=32.04, p<0.01\right)$, Area of Deviation $\left(F_{11,187}=32.05, p<0.01\right)$, and Curvature $\left(F_{11,187}=25.57, p<\right.$ $.01)$. These results and the visual analysis of the mean trajectories (see 1) show that, in some VEs, a significant departure from the shortest trajectory occurred.

Pair-wise comparisons (Bonferroni corrected) between the altered VEs and their baseline VE allow us to identify two sets, depending on the extent of the deviation. The first is the set of those VEs that had a major impact on user trajectories (Objects and Walls); the second set represents those VEs that had a lesser but still significant impact (Water and Dark VEs). The mean trajectories of the VEs in the first set show an almost rectangular shape, with an average deviation of circa $60 \mathrm{~cm}$. This contrasts with the route they performed in the baseline VEs, where they followed the shortest route. The mean trajectories in the second show an average deviation of circa $37 \mathrm{~cm}, 38 \%$ lower. These results are affected by a minority of participants who interpreted the visual conflict in the central area differently and decided to cross over. In practical terms, the minimum deviation values range from the $36.06 \mathrm{~cm}$ in the Water $\mathrm{VE}$ (more than the long size of an A4 sheet) to the $65.23 \mathrm{~cm}$ of the Walls VE (more than the average length of a human arm). Participants also exhibited larger radii of curvature in the altered VEs than in the baseline ones, which indicate straighter paths. Visual inspection of the trajectories indicates that participants were not affected by the order in which VEs were presented. They did not appear to be more likely to adhere to the visual stimuli if they had experienced the stronger alterations (i.e. walls or objects) before the others. Participants were aware of the emptiness of the central area at all times, thus we think the effect is to be ascribed to the design of the VE.

\subsection{Kinematic metrics}

The analysis of kinematic metrics such as Path Length $\left(F_{11,187}=\right.$ $28.72, p<0.01)$, Speed $\left(F_{11,187}=26.00, p<0.01\right)$ and Time $\left(F_{11,187}=20.96, p<0.01\right)$ were also significant. These results supports the stronger effect of the altered indoor VEs. In the Objects and Walls, this resulted in trajectories that were significantly longer in length and were completed more slowly. Indeed, we observed participants moving more carefully around immaterial obstacles, instinctively avoiding collisions. Instead, in the outdoor VEs, the alterations were represented by different surfaces, thus free of potential collisions in the vicinity of the central area.

\subsection{Questionnaires}

The results are summarised in Table 3 . Aside from expected significant differences in terms of presence $\left(F_{9,153}=14.23, p<0.01\right)$ between the real world condition and all the nine VEs, we found a significant difference $(p<0.05)$ between the Ice VE $(M=$ $1.11, S D=1.75)$ and the Walls $(M=3.50, S D=2.07)$ and Dark $(M=3.72, S D=2.44)$ VEs $(p=0.017)$. Indeed participants felt less present in the Ice VE and, although not significant, in the Water VE $(M=1.56, S D=2.25)$. Overall, the indoor VEs were the ones felt most believable, with an aggregated average of 2.77 $(S D=2.38)$, as opposed to $1.74(S D=2.19)$ for the outdoor VEs.

Analogously, no differences were found for the Similarity and Engagement questions, besides those between the real world condi- 

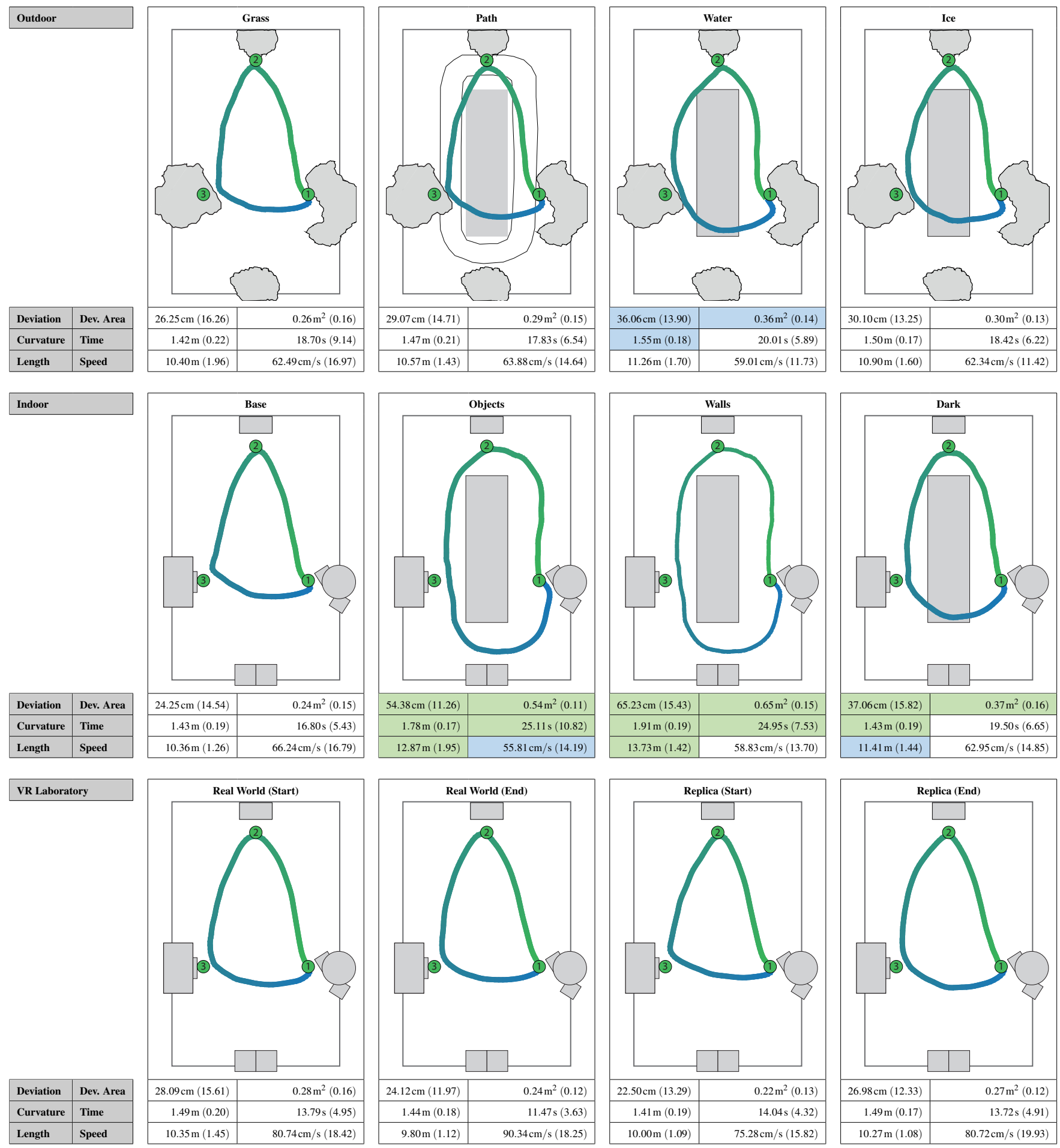

Table 1: In this table, we report the mean results for each type of VEs; in parentheses their respective standard deviation. Significant values, with respect to the baseline environment (the first one on the left), are shown with a blue background if $p<0.05$, with a green background if $p<0.01)$. Trajectories are drawn with a green colour at the beginning, which towards the end progressively becomes blue. The green circled numbers represent the location of the three waypoints. 
tion and the nine VEs. Regarding the question on how much participants felt their movement behaviour was affected, we found a significant difference $(p<0.01)$ between the real world condition $(M=2.33, S D=1.71)$ and all the six altered VEs (aggregated average of $4.52, S D=2.00$ ) not including their respective baseline VE, i.e. Indoor and Outdoor base. However, the score assigned to the Replica VE $(M=3.00, S D=1.46)$ was significantly different $(p<0.01)$ only when compared to the Objects $(M=5.88, S D=1.23)$ and to the Walls $(M=5.83, S D=1.04)$ VEs. This might imply that when the trajectory is altered in a less drastic way (such as in the Water VE), it is harder to notice even though participants were being affected.

\section{Discussion}

Vision is widely known to dominate other senses [8] and VR is no exception [18]. From the moment we don the headset we have to rely on our vision input to make our choices about the environment we find ourselves in. Thus we explicitly placed our participants in situations where they would be subconsciously aware of the conflict between what they saw and what they knew of the real environment. We wanted to better understand the reasoning behind the behaviour our participants exhibited, and how to affect it. In this section we discuss what prompted participants to adhere or refute the visual stimuli. Successively, we present design guidelines and discuss the research challenges that emerged from this study.

\subsection{Adherent Behaviour}

By "adherent behaviour" we mean the set of actions indicating that participants adhered to the visual stimuli, behaving as it would be typically expected under analogous conditions in the real world. For example, walking around a water pond rather than going through it, if not explicitly necessary, or avoiding to walk through a wall, etc. After each environment we interviewed participants on their experience according to what we observed. In the baseline environments (Replica, Outdoor Base, and Indoor Base), all participants took a route close to the shortest one between waypoints, as can be observed by their mean trajectories in Table 1 .

The behaviour of participants who choose to adhere to the visual stimuli can be traced to two main motivations: routine behaviour learnt in past real life situations, and fear of adverse consequences. In the Path scenario we thought that an actual path might suggest a route to follow. We observed four participants keeping to the path. When asked why they did so, participant \#4 commented: "I felt compelled to follow the path. I came from a village like that. I don't know why I followed. I felt I had to." Participant \#13 said: "The path was quite tempting to follow, which I did, partly. I can't explain [why I followed it]." Participant \#14: "I followed the track because it was in the way and it was convenient to follow it." These answers highlight that participants instinctively followed the path and reflects behaviour participants would also adopt in real life.

Participants who decided to walk around the pond, even though that would have increased the length of their route, did so because they were unsure of what would happen if they walked over it. In the water scenario, our quantitative measures indicate that trajectories significantly differ from the most direct one. Participant \#10 said "I avoided the pond because I thought I can't walk across it. I was afraid of walking through it." Similarly, participant \#17: "I walked around the water. I wanted to avoid to get wet." Indeed, without a compelling reason we would probably not step in a water pond: if we just need to go to the other side, we will look for an easier route. These results add to the evidence of people behaving realistically in situations happening in VR [25, 26].

This behaviour conflicts with the lower presence scores assigned to the Water scenario. The majority of participants stated that they did not feel "present" in the VE (with a mean score of 1.56 out of 7). However, the decision of avoiding the water pond points that, during the time of the experiment, the fear of the potential negative consequences was strong enough to overcome their awareness of the illusion. The technical impediments that reinforce the awareness of the simulation, do not impede participants in responding to it realistically. This supports the thesis presented by Slater, who suggests an alternative to the traditional notion of presence in VR [24], consisting of the two concepts of Place Illusion and Plausability Illusion. The former refers to the concept of "being there", whereas the latter is "the illusion that what is apparently happening is really happening (even though you know for sure that it is not)" [25].

Fear of consequences was one of the main reason behind behaviour exhibited in the indoor Objects and Walls scenarios: "You might avoid walking through a wall because there might be a consequence." (\#5); "I didn't want to walk through [the walls]. I wasn't sure what would happen if I went through." (\#6); "I avoided [the objects] because that's what I do in real life." (\#8). Other comments also suggest that interacting with tangible objects mixed with immaterial ones in the same environment, does reinforce the illusion that they might all be tangible: "Because I didn't know which was real and which wasn't so to be sure I decided to walk around." (\#6); "I think having objects you could feel makes you believe that all objects are real." (\#15). One participant commented that "Out of politeness, I didn't touch like I don't in real life." (\#7). Others commented that they wilfully avoided interacting where they were not required: "I don't want to spoil it for myself. I don't want to play around [with the objects]. I want to stay in character." (\#9); "I knew [the wall] wasn't really there and I wanted to respect the environment." (\#11). Thus further research is needed to ascertain whether this behaviour is due to the controlled conditions of the study and whether, in a more comfortable situation (for example, in their own homes), users would be more inclined to discover the boundaries of the system.

In the Ice scenario, we observed only five participants (out of 18) actively trying to walk around the ice. When asked why, the majority of participants did not recognise the icy surface and thought it was rock or even sand. We based our implementation of the ice surface on state of the art real-time shaders. However, it did not evoke our intended outcome. This type of ambiguity was also observed by Simeone et al. [23] where participants had different expectations on a virtual object's temperature or weight based on their interpretation of its material.

Regarding the Dark environment, the majority of participants (11) interpreted it as an unlit floor. However, some were confused over what exactly it was made of: metal, plastic or solid glass over a pit. The remaining seven participants thought that the central area was an unprotected pit and avoided it for that reason: "The centre of the room was like a hole. For self-preservation I didn't want to fall." (\#9). We observed how their behaviour depended entirely on how they interpreted the central area: seven participants took the shortest route because they thought it was solid or were oblivious to any alteration. The rest, afraid of the perceived drop or uncertain of what lay in the middle, decided to avoid it: "It didn't look appealing because of the darkness." (\#4).

\subsection{Non-adherent behaviour}

By "non-adherent" we mean explicitly refusing the visual stimuli by performing actions that in reality would not be possible (such as walking through walls) or improbable (going through a shallow pond to get to the other side, in absence of specific reasons to go into the water). We wanted to understand the factors that prompted participants to act this way, so that they might be addressed. We assume that acting in accordance with the visual stimuli is a desired quality in a VR simulation. If "superhuman" or unrealistic behaviour is desired, then user behaviour should be studied from a different perspective. 


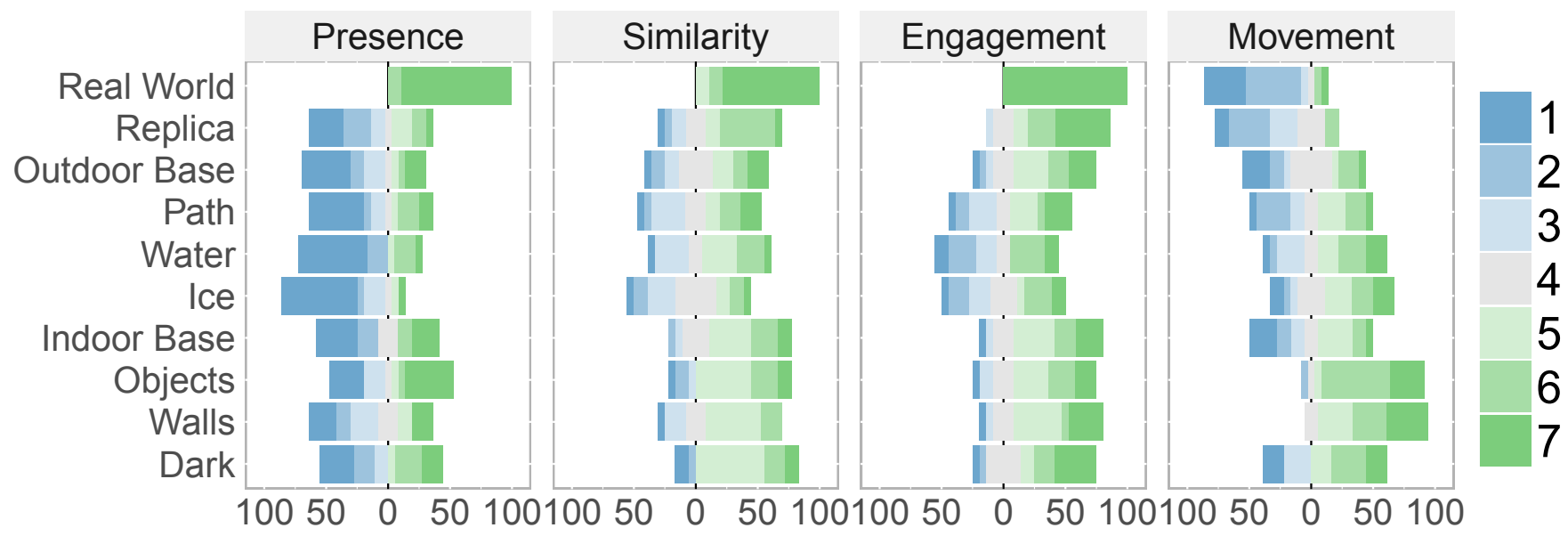

Figure 3: The figure shows the distribution of Presence and Likert scores, grouped by the ten environments. Since the Presence score is calculated as the sum of 6 and 7 responses across the six questions in the SUS questionnaire, scores can range from 0 (dark blue) to 6 (dark green). In the other three plots, responses range from 1 to 7 and are related to the three questions asked: "How similar was your experience of walking in this VE compared to real life", "How engaged were you in the idea of really walking in this VE?", "How much did this VE affect your movement behaviour?".

We observed three main motivations behind non-adherent behaviour: pragmatism, an explicit desire to test the system's boundaries, and the incorrect interpretation of the visual stimuli. In the Path scenario, participant \#9 deviated from the suggested route: "I cut the corner. I think I should be pragmatic at the end." Due to the constraints of a controlled study, there was no apparent benefit in following the path, rather than going directly towards each waypoint. It is thus conceivable that in an actual scenario where the path leads to a specific location, users might be more inclined to follow the path, rather than going through the undergrowth, where rocks or branches might discourage us from following a shorter path.

Other users will attempt to test the boundaries of the system. In the Water scenario we observed some participants tentatively try to step into the pond. Participant \#4 stated: "I walked over the water to see if it reacted." This sentiment was shared by four other participants. When asked "what would make you rethink the idea of stepping into the water?", participants replied that if the water had reacted, through a ripple effect, they would have backtracked. This is in line with considerations made by Slater, that the VR should provide "correlations between external events not directly caused by the participant and his/her own sensations (both exteroceptive and interoceptive)" [25]. However, we add that the VE should also react to events directly caused by the participant.

We observed six participants who attempted to touch the objects or walk through the walls. We asked them why they did so. The common answer was that they wanted to see what it felt like: "I went through to see what would happen. It was uncomfortable for my eyes because I saw total darkness and then I backtracked to the light" (\#17). Three of these six participants went all the way: "I was curious to see what happens when I go through. It was discomforting going inside. I tried to protect myself with my hands” (\#2). The inside area which was walled off was hollow, and the same textures on the visible side were observable from inside. These answers suggest that users might refrain from wilfully colliding with walls once experienced for the first time. One participant tried to rationalise why the immaterial walls were not there: "My brain told me I couldn't touch because I was too far away" (\#4).

Analogously to the Ice and Dark scenario: correct interpretation of the environment is fundamental. We observed two participants that purposely avoided the path surface as they thought it was made out of mud and thus dangerous: "I wanted to climb over the mud.
I asked myself if it is safe or if it was some kind of trap" (\#12). Indeed, they made a conscious effort to avoid placing their feet on the parts of the surface where the path texture was applied. Participants also expected there to be a height difference between the path and the rest of the ground: "The path would have needed me to place my feet on a [lower] height" (\#1).

\subsection{Implications for Design}

After analysing the results of our study and the user behaviour we have extracted a set of guidelines for designers of future VR experiences.

Unambiguous aesthetic alterations can influence user behaviour Our results highlight how it is possible to influence user's movement behaviour by manipulating the appearance of surfaces. The effect we observed on participants' trajectories occurred with their awareness of the physical layout of the room. Surfaces which suggest possible negative consequences if walked upon might be the best suited to achieve this effect, as long as their appearance is unambiguously realistic.

The impact of visual realism in VR is often controversial. There are studies that reported significant differences in terms of behaviour or presence $[26,43,7]$, and those who did not find conclusive evidence $[14,39,46]$. Differently from past work, our study has confronted participants with choices that depended entirely on their correct interpretation of the visual stimuli. We observed completely different behaviour whether participants interpreted the icy surface as such or as something less dangerous such as rock or sand. Thus, we believe that where rendering capabilities are not yet advanced to the point of minimising these ambiguities, it is necessary to supplement visuals with contextual information. For example, through physically responsive VEs (e.g., by showing cracks in the ice once stepped on), intelligent agents [13], or storytelling methods. Future work should investigate whether behaviour is also affected by surfaces whose appearance changes over time, or by moving obstacles. For example, waves on the shoreline, expanding lava flow, etc.

Usage of alterations

Based on our results, we think that alterations to the surface should be used primarily to encourage users to take specific routes. For example, to guide users towards points of interest. Conversely, when the intent is to attempt to block users from moving towards specific locations (such as near fragile items), immaterial objects 
provide a more impactful dissuasion. However, both are passive methods and they should be complemented with active overt methods (such as barriers) as a last resort. For example, when attempting to move outside the tracked area.

Immaterial objects should not present interaction affordances The results support the idea that immaterial objects can have a strong influence on user's movement behaviour. Based on our observations and the frequency with which users attempted interaction with these immaterial objects, we believe those presenting limited or no interaction affordances are those most suited to minimise "breaks in presence" [27]. We chose assets such as tubes or plain walls to delimit the central area in the Objects and Walls VEs. These did not have explicit features that suggested interaction possibilities (such as switches or levers). Thus, these obstacles can be placed in a VE in such a way to guide users towards the areas in the environment that are supported by physical proxies.

Alterations can extend or restrict the space perceived in the VE In the context of VEs that are partly supported by physical objects, alterations to the design of surfaces, and the introduction of immaterial obstacles, allow designers to portray VEs that are larger or smaller than the actual physical environment in which the VR experience takes place. Most participants chose to walk around the obstacles in the Objects and Walls scenarios. This allows designers to introduce immaterial walls to portray narrow hallways in a larger space while maintaining the suspension of disbelief. Conversely, VEs can be extended by using either surfaces that discourage users to walk over them or through immaterial obstacles. For example, a clearing overlooking a cliff, a shoreline, windows, etc.

\subsection{Open Challenges}

The study also allowed us to identify challenges that warrant further research attention, which we highlight in the following.

Transitioning to new locations - In this study we focused on VEs whose extents overlapped with the physical environment. However, this is a limiting factor for multi-location VR experiences. If each location is mapped to the physical environment, future works will need to study forms of transitions between environments that maintain the suspension of disbelief [31]. For example, transitioning from indoors to an outdoor environment larger than the physical room. Novel redirected walking techniques applied to room-scale and cluttered environments could provide a solution.

Synchronisation - In our study, we focused on VEs that are static. However, it is conceivable that through user movement or external agency (virtual agents, events in the narrative), the location of movable objects could be affected. Where objects are paired to physical proxies, this would cause the VE to become out of synch with the physical environment. Tracking the whole physical environment in a domestic setting poses significant technical challenges. Thus, supporting free-form natural interaction in domestic VR will require novel approaches. For example, drones might be used to move in the physical world, an object that moved only in the VE, to re-establish synch.

Height differencs - In the Path scenario, participants commented that they expected the actual footpath to have a lower height than the nearby grass. We know that even relatively small height differences can have a great impact on the suspension of disbelief [11]. However, short of building matching props, replicating height differences becomes difficult in domestic environments. While some objects might lend themselves to support this effect to some extent (such as a rug), further research is needed to provide alternatives allowing users to walk or climb uphill or downhill, in the context of full-body immersive VR system for domestic environments.

\section{Conclusion}

In this paper we investigated how users react when presented with visual stimuli that conflict with their awareness of the physical environment. Knowing how to affect users' movement behaviour can inform the creation of Virtual Environments where users are subtly guided where interaction is supported by tangible elements, or kept away from the boundaries of the tracking area and from fragile objects in the real world. We designed a study asking participants to reach three waypoints arranged in such a way that the shortest route would require them to cross a central area. We manipulated the appearance of this central area in two ways: by altering its aesthetic appearance through different surfaces and by placing different types of immaterial objects.

Quantitative results show that the aesthetic appearance of the surface does influence user trajectories, as long as they are able to correctly interpret its properties. Immaterial objects do also affect user behaviour and were reported to contribute to the believability of the whole experience. User behaviour is affected by perception of negative consequences from experiences in real life and expectations that the Virtual Environment will react to their actions. The study also highlighted three main open challenges: transitions between locations, synchronization between the virtual and physical environment; and simulation of height differences in full-body immersive environments.

\section{REFERENCES}

[1] D. Bowman, D. Koller, L. F. Hodges, et al. Travel in immersive virtual environments: An evaluation of viewpoint motion control techniques. In Proceedings of the Virtual Reality Annual International Symposium, pages 45-52. IEEE, 1997.

[2] G. Bruder, F. Steinicke, and P. Wieland. Self-motion illusions in immersive virtual reality environments. In 2011 IEEE Virtual Reality Conference, pages 39-46. IEEE, 2011.

[3] G. Cirio, A. Olivier, M. Marchal, and J. Pettré. Kinematic evaluation of virtual walking trajectories. IEEE Transactions on Visualization and Computer Graphics, 19(4):671-680, April 2013.

[4] J. Feasel, M. C. Whitton, and J. D. Wendt. Llcm-wip: Low-latency, continuous-motion walking-in-place. In Proceedings of the IEEE Symposium on 3D User Interfaces, pages 97-104. IEEE, 2008.

[5] P. W. Fink, P. S. Foo, and W. H. Warren. Obstacle avoidance during walking in real and virtual environments. ACM Transactions on Applied Perception (TAP), 4(1):2, January 2007.

[6] S. Freitag, D. Rausch, and T. Kuhlen. Reorientation in virtual environments using interactive portals. In Proceedings of the IEEE Symposium on 3D User Interfaces, pages 119-122. IEEE, 2014.

[7] M. Garau, M. Slater, V. Vinayagamoorthy, A. Brogni, A. Steed, and M. A. Sasse. The impact of avatar realism and eye gaze control on perceived quality of communication in a shared immersive virtual environment. In Proceedings of the SIGCHI conference on Human factors in computing systems, pages 529-536, New York, NY, USA, 2003. ACM.

[8] J. J. Gibson. Adaptation, after-effect and contrast in the perception of curved lines. Journal of experimental psychology, 16(1):1, February 1933.

[9] L. F. Hodges, R. Kooper, T. C. Meyer, B. O. Rothbaum, D. Opdyke, J. J. De Graaff, J. S. Williford, and M. M. North. Virtual environments for treating the fear of heights. Computer, 28(7):27-34, July 1995.

[10] E. Hodgson, E. Bachmann, and D. Waller. Redirected walking to explore virtual environments: Assessing the potential for spatial interference. ACM Transactions on Applied Perception (TAP), 8(4):22, December 2008.

[11] B. E. Insko. Passive haptics significantly enhances virtual environments. PhD thesis, University of North Carolina at Chapel Hill, 2001.

[12] V. Interrante, B. Ries, and L. Anderson. Seven league boots: A new metaphor for augmented locomotion through moderately large scale immersive virtual environments. In Proceedings of the IEEE Symposium on 3D User Interfaces. IEEE, 2007. 
[13] M. Luck and R. Aylett. Applying artificial intelligence to virtual reality: Intelligent virtual environments. Applied Artificial Intelligence, 14(1):3-32, 2000.

[14] K. Mania and A. Robinson. The effect of quality of rendering on user lighting impressions and presence in virtual environments. In Proceedings of the 2004 ACM SIGGRAPH international conference on Virtual Reality continuum and its applications in industry, pages 200-205, New York, NY, USA, 2004. ACM.

[15] R. P. McMahan, D. Bowman, D. J. Zielinski, R. B. Brady, et al. Evaluating display fidelity and interaction fidelity in a virtual reality game. IEEE Transactions on Visualization and Computer Graphics, 18(4):626-633, April 2012.

[16] C. Norden. Playstation VR: Development and innovations, 2013. Game Developers Conference (GDC).

[17] T. C. Peck, H. Fuchs, and M. C. Whitton. Evaluation of reorientation techniques and distractors for walking in large virtual environments. IEEE Transactions on Visualization and Computer Graphics, 15(3):383-394, May-June 2009.

[18] S. Razzaque, Z. Kohn, and M. C. Whitton. Redirected walking. In Proceedings of EUROGRAPHICS, volume 9, pages 105-106, 2001.

[19] S. Razzaque, D. Swapp, M. Slater, M. C. Whitton, and A. Steed. Redirected walking in place. In ACM International Conference Proceeding Series, volume 23 of $E G V E$ '02, pages 123-130. Eurographics Association, 2002.

[20] R. A. Ruddle and S. Lessels. The benefits of using a walking interface to navigate virtual environments. ACM Transactions on ComputerHuman Interaction (TOCHI), 16(1):5, April 2009.

[21] R. A. Ruddle, E. Volkova, and H. H. Bülthoff. Learning to walk in virtual reality. ACM Transactions on Applied Perception (TAP), 10(2): 11, June 2013

[22] A. L. Simeone. Substitutional Reality: Towards a research agenda. In Proceedings of the 1st Workshop on Everyday Virtual Reality (WEVR), pages 19-22. IEEE, March 2015.

[23] A. L. Simeone, E. Velloso, and H. Gellersen. Substitutional Reality: Using the physical environment to design virtual reality experiences. In Proceedings of the 33rd Annual ACM Conference on Human Factors in Computing Systems, pages 3307-3316, New York, NY, USA, January 2015. ACM.

[24] M. Slater. How colorful was your day? why questionnaires cannot assess presence in virtual environments. Presence: Teleoperators and Virtual Environments, 13(4):484-493, August 2004.

[25] M. Slater. Place illusion and plausibility can lead to realistic behaviour in immersive virtual environments. Philosophical Transactions of the Royal Society B: Biological Sciences, 364(1535):3549-3557, November 2009.

[26] M. Slater, P. Khanna, J. Mortensen, and I. Yu. Visual realism enhances realistic response in an immersive virtual environment. Computer Graphics and Applications, IEEE, 29(3):76-84, May 2009.

[27] M. Slater and A. Steed. A virtual presence counter. Presence, 9(5):413-434, October 2000.

[28] M. Slater, A. Steed, and M. Usoh. The virtual treadmill: A naturalistic metaphor for navigation in immersive virtual environments. In M. Gbel, editor, Virtual Environments 95, Eurographics, pages 135148. Springer Vienna, 1995.

[29] M. Slater, M. Usoh, and A. Steed. Taking steps: The influence of a walking technique on presence in virtual reality. ACM Transactions on Computer-Human Interaction, 2(3):201-219, September 1995.

[30] J. L. Souman, P. R. Giordano, M. Schwaiger, I. Frissen, T. Thümmel, H. Ulbrich, A. D. Luca, H. H. Bülthoff, and M. O. Ernst. Cyberwalk: Enabling unconstrained omnidirectional walking through virtual environments. ACM Transactions on Applied Perception (TAP), 8(4):25, December 2011.

[31] F. Steinicke, G. Bruder, K. Hinrichs, M. Lappe, B. Ries, and V. Interrante. Transitional environments enhance distance perception in immersive virtual reality systems. In Proceedings of the 6th Symposium on Applied Perception in Graphics and Visualization, pages 1926. ACM, 2009.

[32] F. Steinicke, G. Bruder, J. Jerald, H. Frenz, and M. Lappe. Analyses of human sensitivity to redirected walking. In Proceedings of the 2008 ACM symposium on Virtual reality software and technology, pages
149-156. ACM, 2008.

[33] F. Steinicke, G. Bruder, J. Jerald, H. Frenz, and M. Lappe. Estimation of detection thresholds for redirected walking techniques. IEEE Transactions on Visualization and Computer Graphics, 16(1):17-27, January-February 2010.

[34] F. Steinicke, G. Bruder, L. Kohli, J. Jerald, and K. Hinrichs. Taxonomy and implementation of redirection techniques for ubiquitous passive haptic feedback. In Proceedings of the International Conference on Cyberworlds, pages 217-223. IEEE, 2008.

[35] E. Suma, G. Bruder, F. Steinicke, D. M. Krum, M. Bolas, et al. A taxonomy for deploying redirection techniques in immersive virtual environments. In Virtual Reality Short Papers and Posters (VRW), 2012 IEEE, pages 43-46. IEEE, 2012.

[36] E. Suma, S. L. Finkelstein, M. Reid, S. V. Babu, A. C. Ulinski, L. F Hodges, et al. Evaluation of the cognitive effects of travel technique in complex real and virtual environments. IEEE Transactions on Visualization and Computer Graphics, 16(4):690-702, July-August 2010.

[37] J. N. Templeman, P. S. Denbrook, and L. E. Sibert. Virtual locomotion: Walking in place through virtual environments. Presence, 8(6):598-617, December 1999.

[38] M. Usoh, K. Arthur, M. C. Whitton, R. Bastos, A. Steed, M. Slater, and F. P. Brooks Jr. Walking $;$ walking-in-place $i$ flying, in virtual environments. In Proceedings of the 26th Annual Conference on Computer Graphics and Interactive Techniques, pages 359-364, New York, NY, USA, 1999. ACM Press/Addison-Wesley Publishing Co.

[39] V. Vinayagamoorthy, A. Brogni, M. Gillies, M. Slater, and A. Steed. An investigation of presence response across variations in visual realism. In Proceedings of the 7th Annual International Presence Workshop, pages 148-155, 2004.

[40] M. C. Whitton, J. V. Cohn, J. Feasel, P. Zimmons, S. Razzaque, S. J. Poulton, B. McLeod, and F. P. Brooks Jr. Comparing ve locomotion interfaces. In Proceedings of IEEE Virtual Reality, pages 123-130, Washington, DC, USA, 2005. IEEE.

[41] B. Williams, S. Bailey, G. Narasimham, M. Li, and B. Bodenheimer. Evaluation of walking in place on a wii balance board to explore a virtual environment. ACM Transactions on Applied Perception, 8(3):19:1-19:14, August 2011.

[42] B. Williams, G. Narasimham, B. Rump, T. P. McNamara, T. H. Carr, J. Rieser, and B. Bodenheimer. Exploring large virtual environments with an hmd when physical space is limited. In Proceedings of the 4th Symposium on Applied Perception in Graphics and Visualization, pages 41-48, New York, NY, USA, 2007. ACM.

[43] I. Yu, J. Mortensen, P. Khanna, B. Spanlang, and M. Slater Visual realism enhances realistic response in an immersive virtual environment-part 2. Computer Graphics and Applications, IEEE, 32(6):36-45, November 2012.

[44] C. Zanbaka, B. C. Lok, S. V. Babu, A. C. Ulinski, L. F. Hodges, et al. Comparison of path visualizations and cognitive measures relative to travel technique in a virtual environment. Visualization and Computer Graphics, IEEE Transactions on, 11(6):694-705, November 2005.

[45] D. J. Zielinski, R. P. McMahan, and R. B. Brady. Shadow walking: An unencumbered locomotion technique for systems with under-floor projection. In Proceedings of IEEE Virtual Reality, pages 167-170. IEEE, 2011.

[46] P. Zimmons and A. Panter. The influence of rendering quality on presence and task performance in a virtual environment. In Proceedings of IEEE Virtual Reality, pages 293-294. IEEE, 2003. 